Nouvelles perspectives en sciences sociales

\title{
Edgar Morin et Jesús Ibáñez : sociologie et théories de la complexité
}

\section{Alvaro Malaina}

Volume 3, numéro 2, mars 2008

URI : https://id.erudit.org/iderudit/602475ar

DOI : https://doi.org/10.7202/602475ar

Aller au sommaire du numéro

\section{Éditeur(s)}

Prise de parole

ISSN

1712-8307 (imprimé)

1918-7475 (numérique)

Découvrir la revue

Citer cet article

Malaina, A. (2008). Edgar Morin et Jesús Ibáñez : sociologie et théories de la complexité. Nouvelles perspectives en sciences sociales, 3(2), 9-39.

https://doi.org/10.7202/602475ar
Résumé de l'article

L'article prend appui sur les théories dites « de la complexité » et sur les tentatives d'application de celles-ci à la sociologie. Il compare les perspectives théoriques d’Edgar Morin et du sociologue espagnol Jesús Ibáñez, qui se réclament du " paradigme de la complexité ", en analysant leurs convergences et divergences, en vue de l'élaboration d'une " sociologie complexe». A partir d'une approche comparative, l'article propose des pistes et des orientations possibles pour la construction d'une « science sociale complexe " qui intégrerait une ontologie, une épistémologie et une éthique, et qui devrait également s'ouvrir, sans perdre un esprit critique, aux modélisations contemporaines de la complexité au moyen de techniques de recherche telles que la simulation multiagents. 


\title{
Edgar Morin et Jesús Ibáñez : sociologie et théories de la complexité
}

\author{
Alvaro malaina \\ CETSAH, EHESS de Paris \\ Facultad de Ciencias Políticas y Sociología \\ Universidad Complutense de Madrid \\ Boursier du Ministère Espagnol d'Éducation \\ "Sujet et objet sont indissociables." \\ Edgar Morin \\ "Un physicien est un morceau de matière qui étudie la matière. \\ Un biologiste est un morceau de vie qui étudie la vie. \\ Un sociologue est un morceau de société qui étudie la société. \\ Ce sont tous des miroirs que l'univers place à son centre. " \\ Jesús Ibáñez
}

\section{Introduction : Edgar Morin et Jesús Ibáñez}

Comment situer la démarche d'Edgar Morin, penseur de la complexité et de la transdisciplinarité, par rapport à d'autres démarches contemporaines, voisines ou apparentées? Nous pouvons confronter la perspective de Morin à celle d'un sociologue espagnol qui essaya, lui aussi, d'importer les nouvelles théories de la complexité dans les sciences sociales (de cette façon, nous pourrons complexifier la complexité en suivant la devise de Niklas Luhmann, " la complexité réduit la complexité ») : c'est le cas de Jesús Ibáñez (1928-1992), qui fut directeur d'études à l'Université Complutense de Madrid.

Certains n'hésitent pas à qualifier Ibáñez de "Morin espagnol ", même si sa trajectoire intellectuelle est très personnelle et à vrai dire 
inclassable. Né en 1928 en Cantabrie, il étudie à Madrid, participe aux luttes antifranquistes, notamment à la fondation du Front de Libération Populaire Espagnol (FELIPE) et est expulsé de l'Université en 1956 avec d'autres étudiants, en raison de leur appui aux professeurs renvoyés par le régime dictatorial : Enrique Tierno Galván, José Luis Aranguren et Agustin García Calvo. Après plusieurs mois en prison, profondément insatisfait d'une sociologie académique conservatrice et technocratique, il erre pendant plus de vingt ans dans le secteur privé et introduit, en 1964, le "groupe de discussion " comme technique sociologique d'exploration des représentations des consommateurs. Il fonde l'Institut de Recherches de Marché ECO. Avec l'ayènement de la démocratie, et vivement encouragé par ses collègues, il retourne à l'Université et, en 1982, il devient directeur d'études à la Faculté de Sciences Politiques et Sociologie de l'Université Complutense de Madrid. Considéré comme l'un des intellectuels espagnols les plus charismatiques, il développa, après son retour dans le milieu universitaire et jusqu'à sa mort prématurée en 1992, une sociologie critique très particulière, en opposition à ce qu'il appelait la " sociologie sédentaire".

Morin et Ibáñez sont deux exemples d'un nomadisme permanent, d'une insoumission face aux structures académiques et intellectuelles fixes et que nous pouvons juger endogamiques : deux outsiders qui habitent un no man's land. Les démarches convergent. Morin était, pour Ibáñez, le fabriquant de "la carte de toutes les cartes ${ }^{1} »$. Pour lui, il n'y avait pas de meilleur exercice que de lire l'œuvre de Morin, La Méthode, pour actualiser ses connaissances. "Tout est la ${ }^{2}$ », disait-il. Ibáñez tenta une aventure intellectuelle très similaire à celle de Morin, convergente car elle mobilise les apports des théories de la complexité, mais aussi divergente en raison de son utilisation d'autres sources théoriques telles que le structuralisme, le post-structuralisme et la psychanalyse lacanienne. Face au paradigme classique, il construisit un " paradigme complexe de l'investigation sociale ", en intégrant horizontalement trois perspectives (distributive, structurale et dialectique) et verticalement

Jesús Ibáñer, Más allá de la sociologia. El grupo de discusionn : técnica y crítica, Madrid, siglo XXI, 1979, p. 362.

2 Jesús Ibáñez (coord.), Nuevos avances en la investigacion social l. La investigación social de segundo orden, Barcelona, Proyecto A Ediciones, 1988, p. 173. 
trois niveaux (technologique, méthodologique et épistémologique) ${ }^{3}$. À partir des travaux de Heisenberg, de Gödel, de Spencer-Brown, de von Foerster, de Thom, de Mandelbrot, entre autres, il envisagea finalement une " investigation sociale du second ordre", d'essence fortement critique, face au positivisme dominant et, à certains égards, conservateur.

Il y a de notables convergences entre les travaux de Morin et ceux d'Ibáñez. Tous deux ont défini le système anthropo-social comme un système ouvert, actif et observant, composé par le sujet et par l'objet. Le système existe moins dans la nature que dans l'esprit du sujet qui le conceptualise. Tout système se constitue par le tracé d'une frontière qui le sépare d'un milieu; il est le résultat d'une «ponctuation » qui combine une "distinction", le tracé de la frontière séparant deux côtés, et une " indication » en raison de la préférence pour l'un des deux côtés de celle-ci. Les travaux de Heinz von Foerster et de George SpencerBrown sur l'auto-référentialité paradoxale ont permis à Morin et à Ibáñez de bâtir une forme d'épistémologie constructiviste. Nous y trouvons aussi une ontologie, dans laquelle convergent également leurs points de vue. Le système anthropo-social est un système qui s'autoorganise en autonomie/dépendance par rapport à un milieu ou écosystème, en fonction du bruit, du désordre. L'« ordre par fluctuations » d'Ilya Prigogine et le principe de " complexité par le bruit » d'Henri

3 Le niveau technologique décrit et prescrit «comment se fait ». Le niveau méthodologique demande "pourquoi cela se fait ainsi » et correspond à la sémantique du savoir. Le niveau épistémologique demande "pour quelle raison et pour qui cela se fait ainsi » et correspond à la pragmatique du pouvoir. Chacune des perspectives croise chacun des niveaux : la perspective distributive croise le niveau technologique, la perspective structurale croise le niveau méthodologique, la perspective dialectique traverse le niveau épistémologique. Le modèle de la perspective distributive est le questionnaire; le modèle de la perspective structurale est le groupe de discussion; le modèle de la perspective dialec tique est la socia nalyse (voir Jesús Ibáñez, Del algoritmo al sujeto. Perspectivas de la investigación social, Madrid, siglo XXI, 1985). Même si, en ce début de XXIe siècle, peu d'attention est accordée à certains modèles de recherche (tels que la socianalyse), il est important de noter comment Ibáñez élabora, à l'époque où Morin construisait sa Méthode, un paradigme complexe pour la sociologie, à la lumière des théories de la complexité, mais aussi du structuralisme et de la psychanalyse lacanienne. Nous verrons comment Ibáñez fait correspondre son paradigme avec l'essor de la "cybernétique du second ordre ", face au paradigme classique qui correspondrait à la « cybernétique du premier ordre". 
A tlan les ont influencés. Bien que plusieurs décennies se soient écoulées depuis que Morin et Ibáñez ont proposé un « nouveau paradigme complexe " pour les sciences en général, et les sciences sociales en particulier, ce paradigme a conservé son caractère novateur et son application nous apparaît comme étant toujours nécessaire dans les pratiques de recherche et dans leur cadre théorique. Il est toujours possible d'esquisser une sociologie «complexe » à partir de leurs propos. À ce sujet nous devons tenir compte du fait que les réflexions de Morin et d'Ibáñez ont lieu à une époque déterminée, c'est-à-dire entre 1975 et 1985, au moment où les "sciences » de la complexité deviennent un "paradigme » de la complexitét. On assiste alors à la fondation du paradigme complexe, à partir d'un dialogue fécond auquel participent également, parmi d'autres auteurs, Francisco Varela, Henri Atlan et Jean-Pierre Dupuy ${ }^{5}$. Cependant, nous assistons aujourd'hui à l'incarnation ${ }^{6}$ de toutes ces idées au sein de techniques diverses. Une complexité « restreinte » a pris la place d'une complexité «généralisée $e^{7}$ ». La technique " complexe » par excellence serait celle des automates cellulaires et de la simulation multi-agents, c'est-à-dire des modèles mathématiques modélisés par ordinateur ${ }^{8}$. Nous devons penser à une possible intégration des « deux » complexités. La sociologie complexe ne peut pas être réduite à une philosophie sociale complexe, mais doit être une science sociale complexe à part entière. Cependant, l'intégration est incertaine : si la complexité généralisée manque d'applications

4 Jean-Louis Le Moigne, "Complexité, dans Dominique Lecourt, Dictionnaire d'bistoire et philossopbie des sciences, Paris, PUF, 2006, p. 242.

5 Voir Paul Dumouchel et Jean-Pierre Dupuy, L'anto-organisation : de la pbysique an politique (actes du Colloque de Cerisy, 10-17 juin 1981), Paris, Seuil, 1983.

Jean-Pierre Dupuy, "Les techniques de l'auto-organisation ", Cornelius Castoriadis (1922-1997). Réinventer l'autonomie, $1^{\mathrm{cr}}$ mars 2007, Université Paris VIII.

7 Edgar Morin, «Complexité restreinte, complexité générale ", dans Jean-Louis Le Moigne et Edgar Morin, Intelligence de la complexité. Épistémologie et pragmatique, Paris, Éditions de l'Aube, 2007, p. 28-64.

" Voir Nigel Gilbert et Klaus G. Troitzsch, Simulation for the Social Scientist, Open University Press, McGraw-Hill, 2005. 
techniques, la spéculation théorique fait, en partie, défaut à la complexité restreinte?

Néanmoins, nous trouvons aussi des divergences assez prononcées entre les deux auteurs. Ibáñez, à la différence de Morin, mobilisa le cadre théorique du structuralisme (surtout Lévi-Strauss et Lacan) pour bâtir son paradigme complexe : il fut, en fait, toujours tenté par l'approche structuraliste, bien qu'il ait évolué postérieurement vers un post-structuralisme (Deleuze, Guattari, Foucault, Baudrillard, etc.) et envisagé la dialectique comme horizon final de la science et de la pensée. Nous trouvons également des divergences sur le terrain politique, celui de la praxis du paradigme complexe. Morin, membre du Parti Communiste pendant la Résistance, en fut exclu en 1951. Ibáñez, plus radical, identifié à un marxisme libertaire, resta toujours fidèle à une gauche "radicale", au sens étymologique : rattachée à la racine même de la gauche et non dénaturée. Dans le contexte de cette orientation politique, et tout en s'appuyant sur les théories de la complexité, il fit une critique radicale de la sociologie, en proposant de substituer une "sociologie socialiste » à une "sociologie sociologique ». Ces divergences théoriques concernant le structuralisme deviendront des complémentarités. Ces apparentes divergences politiques concernent plus la forme du discours que le fond. Tous deux croient au changement, à la mutation possible et nécessaire du système anthropo-social qui se dirigerait vers le désastre, mais ils l'expriment d'une façon différente, voire opposée, ce qui peut porter à confusion. Ibáñez est plus rattaché à une pensée faite de catégories et d'oppositions (fondamentalement l'opposition entre les classes dominantes et les classes dominées, entre la majorité et la minorité, entre ceux d'en haut et ceux d'en bas) et finalement à une pensée qui semblerait moins « complexe » que celle de Morin, laquelle serait plus attentive aux nuances, aux ambiguités et aux ambivalences (face au principe d'opposition/disjonction, il propose un principe " dialogique " de distinction/conjonction). Il faut alors tenir

" En France, une tentative de ce genre est formulée dans la récente constitution du réseau thém atique Sociologie et systèmes complexes de l'Association Française de Sociologie (AFS). Voir aussi à ce sujet: Pascal Roggero, "Pour une sociologie d'après La Méthode ", Communications, n 82, Paris, EHESS-Seuil, 2008, p. 143-159 ; Pascal Roggero et Christophe Sibertin-Blanc, "Préambule", Nouvelles perspectives en sciences sociales, vol. $2, \mathrm{n}^{\circ} 2$, Sudbury, Prise de parole, 2007. 
compte du contexte sociohistorique de chacun. Morin forge sa pensée dans un contexte sociopolitique français de démocratie, Ibáñez le fait dans un contexte sociopolitique espagnol de dictature. Ils ne peuvent donc pas mettre l'accent sur les mêmes éléments. Par nécessité historique, le discours d'Ibáñez devait être plus militant, plus combatif, tandis que celui de Morin, moins impulsé par l'urgence du changement, pouvait être plus relaxé. Examinons d'abord les convergences entre eux.

\section{Convergences entre Edgar Morin et Jesús Ibáñez}

Il nous semble possible de caractériser ces convergences, d'abord sur le plan ontologique, puis sur celui de l'épistémologie.

\section{En ontologie}

La complexité peut être comprise d'une façon subjective, comme mesure de l'ignorance du sujet observateur, ou elle peut aussi être comprise d'une façon objective, comme qualité inhérente à l'objet observé. Quels que soient les points de vue, ils sont complémentaires : seul un sujet peut concevoir une complexité objective, seul un objet peut induire une conceptualisation complexe (la perspective complexe se trouve à mi-chemin du subjectivisme et de l'objectivisme, du réalisme et du solipsisme). Nous pouvons ainsi lier une "ontologie " à une "épistémologie ", sans pouvoir les séparer, à partir d'un principe fondamental de relation sujet-objet.

Au niveau de description "ontologique ", la société humaine serait, d'après Morin, un système auto-éco-re-organisé. Plus exactement, il s'agirait d'un système complexe auto-éco-re-organisé. En rassemblant toutes les notions moriniennes et en exploitant leurs applications possibles tout en introduisant des éléments utopiques, nous pourrions aussi l'appréhender comme un système (virtuellement) hypercomplexe auto-éco-re-organisé. La sociologie complexe doit s'édifier en partant de cette notion ${ }^{10}$. Ibáñez définit, à son tour, la société en tant que système

11" - Deux paradigmes fondamentaux aboutissent à la définition morinienne du système anthropo-social. Un paradigme proprement physique (voir Edgar Morin, La Méthode I. La nature de la nature, Paris, Seuil, 1977) : le paradigme tétralogique ordre / désordre / interactions / organisation; et un paradigme proprement biologique (voir Edgar Morin, La Méthode II. La nie de la vie, Paris, Seuil, 1980) : le paradigme de l'auto- 
ouvert, "ouvert au changement", qui «se reproduit en changeant ${ }^{11}$ " et qui établit des limites extérieures (avec le milieu naturel) et des limites intérieures (avec d'autres parties du système) ${ }^{12}$. C'est pour lui un système organisationnellement fermé et informationnellement ouvert ${ }^{13}$. C'est un système hypercomplexe qui inclut des systèmes hypercomplexes (les hommes) ${ }^{14}$. Les ontologies sociales de Morin et d'Ibáñez sont isomorphes et nous pouvons les unifier dans le modèle général de l'autoéco-re-organisation sociale. Un système « organisationnellement fermé» est un système "auto-organisé ", un système "informationnellement ouvert » est un système "éco-organisé ", un système "qui se reproduit en changeant " est un système " té-organisé ».

Il y aurait trois ordres ou niveaux évolutifs du réel : l'ordre physique, l'ordre biologique et l'ordre anthropo-social. Chaque ordre constitutif du réel - et tout ordre sera pensé à partir du troisième ordre, l'ordre anthropo-social - devrait être pensé en s'appuyant davantage sur la notion de "système " que sur celle d'“ objet ", plus rattachée au paradigme précédent. Un système est un ensemble d'interactions entre des éléments différenciés, qui fait émerger une organisation. L'organisation est l'expression de la complexité au sein du système et implique à la fois les idées d'interactions locales et d'émergence globale. Chaque ordre est le produit d'une immersion plus poussée dans la complexité par rapport à l'ordre antérieur, mais la hiérarchie des trois ordres est enchevêtrée. Les trois ordres sont reliés au sein d'une boucle où ils entrent constamment en interaction. La science classique, qui suit le « paradigme de

(géno-phéno-égo)-éco-re-organisation (computationnelle, informationnelle, communicationnelle). Tous deux définiront le paradigme sociologique, ou plus exactement "anthropo-sociologique " (voir Edgar Morin, La Méthode V. L'bumanité de l'bumanité, Paris, Seuil, 2001). Les trois paradigmes s'entrecroisent. Leur caractéristique commune est leur propriété organisationnelle, une propriété émergente qui naît des interactions multiples entre une grande variété d'éléments différenciés, et qui constitue le trait majeur de la complexité.

11 Ibáñez, Jesús, El regreso del sujeto. La investigación social de segundo orden, Madrid, siglo XXI, 1994, p. 29.

2 Jesús Ibáñez, Del algoritmo..., op. cit., p. 191.

13 Jesús Ibáñez (coord.), Nuevos avances..., op. cit., p. 8.

14 Jesús Ibáñez, Del algoritmo alsujeto. Perspectivas de la investigación social, Madrid, siglo XXI, 1985 , p. 78. 
la simplification ", selon l'expression morinienne, les conçoit séparément. Mais dans la réalité ils sont inséparables et une nouvelle science - qui suivrait le "paradigme de la complexité »- devrait les concevoir et les traiter ensemble ${ }^{15}$. La pensée complexe serait capable de penser à la fois les niveaux successifs, autonomes, mais reliés, sans les séparer ni les réduire. Une pensée qui opère au niveau des seuils et des frontières entre les différents ordres émergents. Une pensée qui relie, qui unit ce qui est "par nature » lié et joint (com-plexus). Le réel est un " espace lisse » transformé en "espace strié ${ }^{16}$ " par le regard de l'observateur, voire du scientifique, regard qui vise in fine la manipulation du réel. L'espace lisse est un espace isotropique, sans barrières ni frontières ni chemins préétablis, tandis que l'espace strié est un espace anisotropique, avec des barrières, des frontières et des chemins préétablis. Le paradigme classique postule une science pour chaque ordre, et aucune communication transversale entre les différentes sciences. Mais une science complexe et non classique ne devrait pas se réduire exclusivement au modèle de l'espace strié - en prenant en compte uniquement les singularités et les spécificités de chaque ordre -, mais s'approcher de plus en plus du modèle d'espace lisse - prenant également en compte la liaison et les communications entre les différents ordres--, en mettant en ceuvre une transdisciplinarité (Morin) et un nomadisme (lbáñez) permanents : une communication rhizomatique, c'est-à-dire dans tous les sens et dans toutes les directions. C'est la seule façon d'articuler une approche effective, bien que toujours asymptotique (Lacan), du réel un et multiple. Ibáñez, se revendiquant des mêmes sources scientifiques que Morin, décrit, lui aussi, le réel en tant que structuré par trois strates fondamentales : la strate physique, la strate vitale et la strate psycho-sociale. Le concept de "strate " peut être remplacé par le concept de «système ». La strate évoquerait l'idée de multiplicité et d'ouverture, alors que le système serait associé à l'idée d'unité et de fermeture ${ }^{17}$. Les strates composent des êtres individualisés. Elles composent des ordres organisationnels : physique, vital, psycho-

15 Edgar Morin, La Métbode I..., op. cit., p. 19.

16 Voir Gilles Deleuze et Félix Guattari, Capitalisme et schizopbrénie : mille platetanx; Paris, Minuit, 1980.

17 Jesús Ibáñez, Del algoritmo al sujeto. ..., op. cit. p. 131. 
social. La complexité organisée augmente au fur et à mesure que nous évoluons de strate/système en strate/système. Il importe de distinguer la complexité organisée de la simplicité organisée (systèmes mécaniques) et de la complexité désorganisée (systèmes stochastiques). La complexité organisée est un " réseau complexe de relations entre éléments, c'est-àdire une structure, et un processus complexe de telations entre relations, $c^{\prime}$ est-à-dire un système ${ }^{18}$ ». Pour Anthony Wilden, l'une des influences principales d'Ibáñez réside en ce qu'un ensemble, pour former un système complexe organisé, doit comprendre des éléments, une structure (relation entre éléments) et un système (relations entre relations) ${ }^{19}$. La simplicité organisée ne comporte que des éléments et une structure; la complexité désorganisé n'est constituée que d'éléments et d'un système ; la complexité organisée réunit les trois composants : éléments, structure et système.

Le système anthropo-social fait partie de la biosphère; il est composé par des êtres vivants, les hommes. En tant que phénomène organisationnel, il possède les mêmes caractères que tout phénomène organisationnel vivant, en constituant un être ${ }^{20}$. Son organisation propre (Morin parle d'" organisations actives") est une auto-organisation (émergence organisationnelle) : le système social s'auto-produit et s'auto-extériorise à partir des interactions des individus ${ }^{21}$. Parallèlement,

18 Ibid., p. 177.

19 Anthony Wilden, System and Structure. Essays in Communication and Exchange, Londres, Tavistock, 1972, p. 204.

20 «L'idée d'être n'est pas une notion substantielle. C'est une idée organisationnelle... il y a émergence d'être là où il y a organisation " (Edgar Morin, La Méthode I. La nature de la nature, Paris, Seuil, 1977, p. 211).

21 Le sujet par ailleurs « controversé » de l'auto-extériorisation du social a été bien traité par Jean-Pierre Dupuy (voir Introduction aux sciences sociales. I ogique desphénomènes collectifs, Paris, Ellipses, 1992) à travers l'analyse des discours libéraux qui se réclament de l'auto-organisation du social. L'exemple le plus connu est celui de Friedrich von Hayek, qui, tout en s'appuyant sur la vague cybernétique, opposa l'« ordre social spontané" (qui naît des actions des hommes, mais pas de leurs desseins) au "constructivisme social" (où l'organisation sociale est issue d'un programme, des desseins des hommes). Le «constructivisme social» conduirait, selon Hayek, à la servitude et aux totalitarismes. Le marché serait en revanche l'exemple parfait de l'« ordre spontané ". À la différence des thèses hayekiennes, on préfère ici concevoir l'autonomie organisationnelle au sens d'une "auto-institution " permanente de la société, idée 
il définit toujours un éco-système (social et naturel), avec lequel il échange de la matière, de l'énergie et de l'information, qui constituent des apports nécessaires de néguentropie qui lui permettent de surmonter le deuxième principe de la thermodynamique ${ }^{22}$. 11 se réorganise et assure en parallèle les conditions de sa permanence et de sa reproduction à travers des boucles de rétroaction positive et négative, reproduction qui s'effectue en changeant (re-production et re-nouvellement). L'organisation propre au système anthropo-social est donc, plus exactement, une auto-éco-re-organisation. Selon Jean-Louis Le Moigne, l'autoorganisation exprime l'autonomie, l'éco-organisation exprime le fonctionnement (synchronique) ouvert dans l'environnement et la reorganisation exprime la transformation (diachronique) ${ }^{23}$.

Il est nécessaire de distinguer entre " complexité " et " hypercomplexité ». Tout système complexe auto-éco-re-organisé combine centrisme, polycentrisme et acentrisme. Un système hypercomplexe " diminue ses contraintes tout en augmentant ses aptitudes organisationnelles, notamment son aptitude au changement ${ }^{24} \%$. Il atténuerait le centrisme et accentuerait le polycentrisme et l'acentrisme. Dans le cas du système anthropo-social, il s'agirait d'un système plus démocratique, c'est-à-dire qui dissémine de plus en plus le pouvoir dans tout le corps social, sans instance centrale et suprême chargée de le gérer. Mais ce modèle, nous le savons bien, est encore utopique ${ }^{25}$, car il prend place

développée par Cornelius Castoriadis et chère à Morin. Cela serait plus en rapport avec l'idée de système téléologique et morphogénique, composé d'agents intentionnels, caractéristique essentielle du système anthropo-social, que nous développerons dans ce qui suit.

22 Mais il faut être prudent. Comme nous le verrons, d'après l'analyse de Wilden, la logique de définition et d'ouverture à un éco-système peut aussi être la logique arbitraire de l'exploitation et de l'asservissement.

23 Jean-Louis Le Moigne, La modélisation des systèmes complexes, Paris, D unod, 1990, p. 74.

24 Edgar Morin, Le paradigme perdu : la nature bumaine, Paris, Seuil, 1973, p. 130.

25 L'idée et l'expérience de l'«utopie » furent très chères à Morin dès la fin des années soixante. On peut le voir dans ses livres Mai 68: I a breche (en collaboration avec Claude Lefort et Cornelius Castoriadis), Paris, Fayard, 1968 et dans Journal de Californie, Paris, Seuil, 1970. Curieusement, ses expériences de Mai 68 et de la Californie marquent d'une certaine façon le début de son voyage en quête de la complexité. Pour Wilden, les années soixante furent la tentative de la part du système de se montrer dans sa globalité : «les années soixante furent l'explosion du contexte de la 
dans le temps, dans un futur possible mais improbable. Pour Ibáñez, plus radical, la société est un système hypercomplexe qui implique le passage d'une "individuation biologique complexe " à une "individuation noologique ou psycho-sociale hypercomplexe ${ }^{26} "$. L'hypercomplexité est, pour lui, liée à une réflexivité du second ordre : " [l] es systèmes sociaux sont hypercomplexes (des systèmes réflexifs - les sociétés - qui incluent des composants réflexifs - les individus : ils opèrent une réflexion du second ordre, quand la société se pense à travers ses penseurs) ${ }^{27}$ \%. L'auto-réflexivité est la condition de base de la démocratie.

Pour Ibáñez, le système anthropo-social est un système linguistique qui est structuré par des codes linguistiques : «[1]es codes génétiques (proto-linguistiques) prédominent dans l'ordre vital, les codes linguistiques (à proprement parler) prédominent dans l'ordre social. Les codes génétiques ont une pragmatique, les codes linguistiques ont en outre une sémantique car ils sont interprétés par un sujet conscient. Les codes génétiques décrivent le système, les codes linguistiques décrivent en outre l'écosystème $e^{28}$ ». Ibáñez précise : « [l]'ordre social est de l'ordre du « dire » : il est fait de dictées (chemins prescrits) et d'interdictions (chemins proscrits) ${ }^{29} \mathrm{n}$. Le système anthropo-social opère non seulement dans le registre lacanien du réel où opèrent exclusivement les soussystèmes biologiques et physiques qui le forment, mais également dans les registres imaginaires et symboliques. C'est un système auto-réflexif : ses agents constitutifs sont des agents auto-réflexifs, cognitifs et intentionnels; son dispositif informationnel/sémiotique de base est un dispositif de lecture et d'écriture. «Les étants physiques n'ont pas besoin de connaître la physique, les étants biologiques connaissent inconsciemment la biologie, les étants sociaux connaissent consciemment la sociologie (ils savent qu'ils savent). Les éléments d'un système linguistique sont réflexifs... Ils sont en outre actifs (leur comportement est 


\section{NPSS, VOLUME3,NUMERO 2, 2008}

intentionnel) ${ }^{30}$ ". Le système anthropo-social est un système dynamique, qui évolue et se complexifie avec le temps. Situé au carrefour de l'aléatoire et du nécessaire, le système anthropo-social, en tant que système naturel, se complexifie par les mutations génétiques, mais en tant que système linguistique, il est aussi l'objet de mutations linguistiques, d'une écriture : le «bruit » dans le système anthropo-social naturel, linguistique et mnémonique est métabolisé en tant que «trace» qui persiste, retransmise par les codes génétiques et linguistiques, et qui deviendra finalement " sens ${ }^{31}$ ". «Un code est un hasard gelé. Les codes sont des dispositifs à double articulation : une seconde articulation émergeant par hasard est retenue par une première articulation (nécessaire) ${ }^{32}$ ". "Le passage de la seconde articulation à la première articulation est le passage de l'énergie à l'information ${ }^{33}$ ". Morin a également construit le couple genos/pbenon ${ }^{34}$; l'auto-organisation sociale serait une auto-(geno-pheno)-éco-re-organisation : elle implique un code, mais aussi une actualisation de ce code dans un environnement, au moyen d'un être et d'une existence individuels. Morin et Ibáñez conçoivent le système anthropo-social comme un système dynamique non linéaire et imprévisible. Il a une histoire (ce qui implique que ce n'est pas seulement l'environnement du système qui change, mais le système luimême, ce qui exige une démarche systémique non classique capable d'étudier à la fois les perturbations externes - outputs - et les fluctuations internes - changements d'état, code ou structure internes), marquée par l'irruption constante d'événements ${ }^{35}$ (Révolution Française, Commune de Paris, Mai 68, chute du Mur de Berlin, attentats du 11 septembre, etc.), qui seraient les bifurcations et les catastrophes inattendues et nécessaires à l'évolution et à la complexification du système anthropo-

3"1 lbid., p. 62 .

31. Mais le bruit ne vient pas de l'extérieur. C'est le produit nécessaire des tensions internes entre les différents sous-systèmes de "l'éco-système historique" (Anthony Wilden, "Context'Theory... ", op. cit., p. 402).

32 Jesús Ibáñez, El regreso..., op. cit., p. 10.

33 Ibid., p. 82.

34 Edgar Morin, La Méthode II..., op. cit., p. 113.

35 Edgar Morin, «L'événem ent-sphynx », Communications, 18, 1972; Jesús Ibáñez,, Más allá..., op. cit., p. 151-157. 
social (rupture de la continuité systémique, rupture de l'ordre interne, absence de continuité et de dérivabilité, apparition de l'anti-organisation au sein de l'organisation, processus sémiotiques/pulsionnels d'écriture). La perspective structurale initiale est surmontée par une perspective dialectique. Le système anthropo-social hypercomplexe auto-éco-reorganisé serait finalement un système dynamique morphogénique ${ }^{36}$, créateur de nouvelles formes. Cela ne va pas sans conséquences politiques, qui semblent dépasser le cadre strictement scientifique. Le système anthropo-social s'auto-produit, évolue et se révolutionne. Il " s'auto-institue » sans cesse comme le montre Castoriadis ${ }^{37}$. Il sécrète des structures et des formes changeantes. Une míse en parallèle avec la démocratie devient alors pertinente. La sociologie complexe que nous sommes en train d'exposer devrait y réfléchir, mais le but de cet article n'est pas de développer davantage ces réflexions : nous ne faisons qu'apporter des pistes et des clés de réflexion. Cependant, nous ne devons pas avoir peur de les formuler : comme nous allons le voir à présent, le sociologue est trop proche de la société qu'il étudie pour croire qu'il peut s'en détacher en faveur d'une "objectivité " présupposée. Il est cependant l'un des acteurs qui appartiennent et donnent forme (en in-formant) au système anthropo-social : par action ou par omission. C'est la seconde dimension réflexive qu'ouvre la pensée de la complexité.

\section{En épistémologie}

Le système se trouve également et, pour de nombreux auteurs, surtout dans l'esprit du systémiste. Nous constatons alors que l'ontologie complexe postulée par Morin et Ibáñez repose sur une épistémologie de base.

Morin et Ibáñez participent au courant de pensée que l'on nomme "cybernétique du second ordre», dont les principaux représentants sont

36 Anthony Wilden (System and Structure.., op. cit., 355 p.) oppose à l'homéostase (la conservation et la reproduction d'une forme spatiale) et à l'homéorhèse (la conservation et la reproduction d'une forme temporelle), la morphogenèse (la production d'une nouvelle forme). Le terme de morphogenèse est employé par Wilden dans un sens analogue à celui où l'emploie René Thom, en tant que restructuration dialectique. La perspective dialectique formulée par Ibáñez vise la morphogénèse sociale.

${ }_{37}$ Cornelius Castoriadis, L'institution imaginaire de la societé, Paris, Seuil, 1975. 


\section{NPSS, VOLUME 3, NUMERO 2, 2008}

Heinz von Foerster, Francisco Varela, Humberto Maturana, Gordon Pask, etc. Ce courant de pensée opère un tournant épistémologique fondamental dans la cybernétique classique et dans la science classique en général. Morin et Ibáñez essaieront de l'appliquer aux sciences sociales. La cybernétique, science des mécanismes de communication et de contrôle, fut inaugurée par Norbert Wiener en 1948 (Cybernetics) et fut fondée sur l'idée de système en tant que machine allopoiétique qui suit un schéma d'entrées et de sorties (inputs et outputs) programmable de l'extérieur. Nous pouvons situer l'annonce de la " nouvelle cybernétique » à l'année 1952, lors de la neuvième conférence de Macy. W. Ross Ashby qui introduit l'« homéostat ", le prototype d'une machine auto-régulée, capable d'ajuster ses fluctuations internes pour s'adapter aux mutations de son environnement. Mais Ashby appartenait encore à la cybernétique classique : il énonce l'impossibilité logique pour un organisme d'accéder à une complète autonomie organisationnelle ${ }^{38}$. Cependant, le renversement s'était déjà opéré : la machine allopoiétique se transforme en machine autopoiétique. Heinz von Foerster fonde « officiellement» la nouvelle cybernétique en créant en 1956 le Biological Computer Laboratory de l'Université d'Illinois et en introduisant en 1960 son concept central d'« ordre à partir du bruit» (order from noise) ${ }^{39}$. Le bruit ne serait destructeur que pour des systèmes simples; au sein des systèmes complexes, il provoque l'apparition d'un ordre, d'une complexité. La machine autopoiétique s'« auto-organise » à partir du bruit et du désordre. Les travaux postérieurs d'Henri Atlan (auto-organisation des systèmes vivants), de Francisco Varela et Humberto Maturana (autopoièse) et d'Ilya Prigogine (structures dissipatives), parmi d'autres, consolideront les fondations du nouveau paradigme cybernétique des systèmes auto-organisés, qui deviendra le nouveau paradigme de la complexité. Ce fut von Foerster qui transforma la cybernétique en épistémologie. La cybernétique classique ou cybernétique du premier ordre était une science de la maitrise et du contrôle. Son principal postulat était celui de l'objectivité. Il y aurait une stricte séparation entre

38 Voir à ce sujet Jean-Pierre Dupuy, Aux origines des sciences cognitives, Paris, La Découverte, 1994.

3" Heinz von Foerster, "On Self-Organizing Systems and their Environments ", SelfOrganizing Systems, New York, Pergamon Press, 1960, p. 31-50. 
le sujet observateur et l'objet observé qui donne accès à la maîtrise. Von Foerster participe en revanche du « constructivisme radical » : l'objet est une construction du sujet. Toute réalité correspond à une sélection et à une distinction (c'est le " dessin d'une différence ", comme le disait Spencer-Brown) et toute sélection et toute distinction sont opérées par un sujet (qui introduit des valeurs, des indications pour chaque côté de la frontière qu'il trace). L'impossibilité de séparer l'objet du sujet implique l'impossibilité du contrôle et de la maitrise de l'objet par le sujet. D'une part, l'objet est une construction du sujet ; d'autre part, l'objet devient sujet, en l'occurrence il devient machine autopoiétique. Sujet et objet font un. La cybernétique des systèmes observés se transforme en cybernétique des systèmes observants. L'observateur fait partie de ce qu'il observe; il est toujours en train de s'observer luimême. Sujet et objet font partie d'un même système observant.

Les révolutions dans le domaine de la mécanique marquent le passage de l'ancien paradigme simplifié au nouveau paradigme complexe. Dans la mécanique classique, le sujet était extérieur à l'objet et pouvait l'observer et l'appréhender : c'était la position du " sujet transcendantal ». Dans la mécanique relativiste, le sujet est «bouleversé " par l'objet : l'observation dépend d'abord du lieu du point de vue de l'observateur ; la position du sujet devient multiple et conditionnée. Dans la mécanique quantique, l'objet est "bouleversé " par le sujet : le sujet observateur influence l'objet observé ; sujet et objet s'interpénètrent; le système observant sujet/objet émerge pour résoudre les paradoxes ${ }^{40}$. Pour bâtir leur épistémologie, Morin et Ibáñez mobilisent les apports fondamentaux de Heisenberg et de Gödel, qui ont contribué à détrôner le sujet transcendantal en substituant au principe classique de l'objectivité, le principe non classique de la réflexivité : nous ne pouvons pas déterminer à la fois la position et la vitesse d'une particule (la vérité en tant que preuve empirique est "déconstruite»); nous ne pouvons pas prouver la vérité de tous les énoncés d'une théorie (la vérité en tant que preuve théorique est « déconstruite »)

4i) Jesús Ibáñez, El regreso..., op. cit., p. 91-92.

41 La psychanalyse nous a montré que la réalité du sujet est la réalité de sa division successive et de sa perte des objets. Tout d'abord au stade du miroir, le sujet se confond avec son reflet fantasmatique. Plus tard, avec l'imposition de l'ordre 


\section{NPSS, VOLUME 3, NUMÉRO 2, 2008}

Un principe fondamental de la relation sujet/objet découle des apports de la cybernétique du second ordre et de la mécanique quantique, et c'est surtout lors de l'extraction de ses implications sociales et sociologiques que la forme, et non pas le contenu, des discours de Morin et Ibáñez diverge.

\section{Divergences entre Edgar Morin et Jesús Ibáñez}

\section{Dans les implications en sociologie de l'onto-épistémologie complexe}

Pour Ibáñez, le système anthropo-social serait une "machine naturelle autopoiétique \%. Elle serait donc démocratique par nature, au sens d'autonome et auto-productrice. Une dictature chercherait en revanche à le transformer en une machine artificielle allopoiétique ou hétéronome et hétéro-produite. Elle serait condamnée à échouer : la nature finit toujours par s'imposer. Selon Ibáñez, qui s'appuie sur les théories de l'autopoièse des biologistes chiliens Francisco Varela et Humberto Maturana, le système anthropo-social serait un système organisationnellement fermé (auto-organisé à partir d'interactions multiples entre des acteurs différenciés) et informationnellement ouvert (réflexif, producteur d'information). Les " classes dominantes" voudraient, en revanche, le transformer en système organisationnellement ouvert (hétéro-organisé de façon univoque, selon leurs desseins et leurs manipulations) et informationnellement fermé (non réflexif, non producteur d'information). Le sociologue classique, positiviste, serait à leur service (d'une façon directe ou indirecte) : il extrairait de l'information des bases (des "classes dominées») pour les sommets, afin que ceux-ci puissent injecter de la néguentropie (in-formation) de haut en bas pour neutraliser toute tendance à la désorganisation et pour maintenir l'ordre social qui sert leurs intérêts. Nous constatons déjà des divergences entre Ibáñez et Morin, qui se manifestent surtout au niveau du langage. Ibáñez se réclame volontiers de la tradition critique

symbolique, le sujet se divise à nouveau : d'une part, le sujet qui énonce, d'autre part, le sujet énoncé. Parallèlement, le sujet commence à expulser des parties de lui-même qui constitucront les «objets». Le réel nous échappe toujours : c'est «l'impossible " (Lacan). Ce que l'on rencontre à sa place, c'est le «masque» (la personne), le fictif (la Réalité), le théâtral (le social). C'est l'invention, la réorganisation permanentes. 
marxiste ; Morin hésite et doute de la pertinence de l'usage de la terminologie marxiste.

Selon Ibáñez, le sociologue positiviste classique, durkheimien, opère une mystification; il croit être le seul sujet, il transforme le système anthropo-social observant en objet : c'est le paradigme classique du fait social considéré comme " chose ". Mais le mot " fait » dérive du latin factum, qui est le participe passé du verbe facere ("faire »). C'est-àdire que le "fait " social est toujours " fait " par quelqu'un. Le sociologue positiviste l'ignore et définit le système (en perturbant sa nature) et trace ses frontières (en opérant des séparations) : il se situe luimême dans l'éco-système. Ce faisant, il contribue au maintien du système anthropo-social dans sa forme actuelle (qu'elle soit juste ou injuste), car il prive le faux objet de sa vraie nature de sujet. De cette façon, insiste Ibáñez (en transformant la sociologie complexe en sociologie critique de la sociologie), la structure hylémorphique (Simondon) se maintient : les "sujets seigneurs " décident et programment (ils apportent la forme), les «objets esclaves » exécutent leurs ordres (ils apportent la matière) ${ }^{42}$. Max Weber corrigea l'objectivisme de Durkheim en proposant une méthodologie herméneutique et compréhensive, capable d'étudier le fait social de l'intérieur du sujet qui l'incarne: or, il conserva le postulat d'une neutralité axiologique de la part du sociologue, ce qui situe sa démarche au mi-chemin d'un paradigme de simplification et d'un paradigme de complexité. La neutralité objectiviste sera toujours dominante. Le sociologue sédentaire (qui fonctionne comme une machine classique avec des idées et des méthodes rigides, ses liens de constitution) adopte le point de vue de la majorité, regarde du haut des hiérarchies, du lieu du pouvoir et de l'unité ; le sociologue nomade (qui fonctionne comme une machine quantique avec des idées et méthodes souples) adopte le point de vue des minorités, pousse de la base de la pyramide, du lieu de la multiplicité qui résiste au pouvoir et à l'unitée ${ }^{43}$. La sociologie sédentaire et dogmatique conserve et reproduit ; la sociologie nomade et critique invente et produit. Il ne s'agit pas de les opposer : ce sont deux moments d'un même processus dynamique, en l'occurrence du niveau

42. Jesús Ibáñez (coord.), Nuevos avances..., op. cit., p. 7.

43 Jesús Ibáñez, Del algoritmo..., op. sit., p. 37. 


\section{NPSS, VOLUME 3,NUMERO 2, 2008}

synchronique de l'éco-organisation et du niveau diachronique de la réorganisation. La sociologie sédentaire est du côté de la morphostase (constance de la forme) ; la sociologie nomade est du côté de la morphogénèse (variation de la forme et création de nouvelles formes). Mais pour pouvoir inventer et produire, il faut traverser la frontière, voile de $\mathrm{Maia}^{44}$, qui sépare le sujet de l'objet.

La critique d'Ibáñez est radicale : elle dépasse le seuil du savoir (la science) en s'aventurant dans le domaine parallèle du pouvoir. Morin, plus tempéré, préfère rester au niveau du savoir, mais tout en visant toujours un hotizon éthique et sans jamais négliger les interrelations entre le scientifique et le social via le paradigme ${ }^{45}$. Même si nous constatons certaines divergences entre les deux - surtout au niveau de ce qu'ils soulignent en priorité -, ces dernières ne sont qu'apparentes. Le savoir et le pouvoir marchent toujours ensemble : tous deux le savent, mais l'expriment de façon différente.

La réalite ${ }^{46}$ devient «construction ${ }^{47}$ ». Le philosophe espagnol Agustín García Calvo distingue entre le réel (la réalité, tout ce qui existe, la physis) et la Réalité (l'artifice opératif anthropomorphique qui vient se substituer au réel et qui correspond à la totalité des choses définies). Tout système anthropo-social, au moyen de dispositifs linguistiques, construit une Réalité. Pour les parties du système, pour les êtres humains, la réalité sera toujours la Réalité (l'objet est perdu) où habiteront leurs «masques" (le sujet est divisé). Mais un constat en découle : au-delà de la Réalité actuelle, nous pouvons, par conséquent, construire des Réalités différentes, même si elles ne sont pas des réalités, ou à cause de

44 C'est là l'objectif final des différents mysticismes orientaux. La séparation entre le sujet et l'objet ne serait qu'une brume illusoire, appelée Maia par les Hindous, qui crée des substances et des oppositions là où il n'y a que des relations et des liaisons.

4.5 Edgar Morin, «Paradigmatologie», La Méthode II’. Les. idées, Paris, Seuil, 1990, p. 211 238.

40 Le "tohu-bohu ", le bruit, le bruit de fond, selon Michel Serres. Chaos, abime, sansfond, selon Cornelius Castoriadis. L'océan primordial du désordre d'où naissent les petits îlots d'ordre où siègent les hommes et les sociétés, c'est-à-dire les systèmes anthropo-sociaux (qui présentent un ordre organisationnel issu du chaos et maintenu à partir d'un code linguistique).

47 Paul Watzlawick (dir.), L'invention de la réalité. Contributions an constructivisme, Paris, Seuil, 1988. 
cela précisément. Dans le cas des Réalités sociales, nous pouvons même construire des Réalités plus justes et appropriées. Nous pouvons définir et créer des Réalités sociales plus justes et appropriées. Pour cela, le processus d'écriture devient fondamental, clé du passage des sociétés «froides", qui ont une structure invariante, aux sociétés « chaudes», qui ont une structure changeante. La société froide est mécanique et artificielle (paradigme de la simplicité organisée), tandis que la société chaude est complexe et naturelle (paradigme de la complexité organisée).

Pour Anthony Wilden, tout système, au moyen du tracé d'une frontière, définit un éco-système où il place la partie de soi-même qu'il cherche à exploiter et qui devient nécessaire à sa survie : source de néguentropie. Par exemple, pour le système anthropo-social, les femmes, les enfants, le milieu naturel, les Noirs ou le Tiers-Monde. Selon Wilden, le système est en guerre avec lui-même. La mystification opérée par le sociologue positiviste devient la mystification d'une forme sociale tout entière qui répond aux intérêts d'une couche sociale particulière (le discours scientifique classique ne serait dans ce sens que "propagande ). Le paradigme classique, celui de la « simplification », est un paradigme de la matière-énergie (c'est-à-dire le corps humain et sa force de travail) ; en revanche, le paradigme non classique, celui de la " complexité ", est un paradigme de l'information (qui transforme la matière inerte en matière vivante, qui fait croître la liberté sémiotique des parties face aux contraintes du tout). L'information qui in-forme et traverse toutes les couches de la réalité - les trois mondes - devrait être placée du côté des «sujets » exploités en tant qu' «objets ${ }^{48}$ ». La voie de l'exploitation de l'éco-système devient une voie auto-destructrice pour le système, car l'exploitation équivaut à l'épuisement d'une source, voire d'une source de néguentropie nécessaire à la survie du système.

La pensée complexe morinienne se fait écho de toutes ces idées révolutionnaires ( Systémisme et cybernétique sont comme le premier étage d'une fusée qui permet le démarrage d'un second étage, la théorie de l'auto-organisation, laquelle à son tour met feu à un troisième étage,

48 L'éco-système n'est pas seulement une source de matière et d'énergie, c'est aussi un centre producteur d'information capable de changer la structure du système. 
épistémologique, celui des relations entre le sujet et l'objet ${ }^{49}$ ») et aboutit aux mêmes conclusions : le sujet ne peut pas être séparé de l'objet, le sociologue ne peut pas être séparé de la société ("sujet et objet sont indissociables $\left.{ }^{50}\right)$ ). Pascal disait : le monde me comprend, mais je le comprends. L'écrivain américain Paul Auster répète souvent le paradoxe : le monde est dans ma tête, tandis que ma tête est dans le monde. Morin est également surpris par la même réflexion : la société est dans le sociologue qui est dans la société $e^{51}$. L'auto-référence s'impose sans délais. La boucle paradoxale a une seule solution logique : concevoir un système qui transcende la séparation entre le sujet et l'objet en les incluant dans une même unité multiple (unitas multiplex).

Mais l'application à la sociologie des théories de la complexité est plus tempérée chez Morin. Nous devons certes « intégrer l'observateur/concepteur (le sociologue) dans son observation et sa conception $^{52}$ ". Cette idée conduit à l'élaboration d'une "sociologie de la sociologie " Cependant, la plus importante différence avec Ibáñez se trouve dans le fait que la critique d'Ibáñez ne se contente pas de préconiser cette insertion du sujet sociologue dans son objet en vertu d'une approche scientifique du réel plus rigoureuse (pour Morin, le sociologue, avant d'étudier les valeurs et la culture d'une société donnée, devrait étudier ses propres valeurs et sa propre culture), il ne se' contente pas de préconiser une sociologie de la sociologie, mais élabore une sociologie critique (des «classes dominantes ", de ceux qui possèdent le pouvoir et maintiennent l'ordre social), qui se transforme en critique de la sociologie (en tant que dispositif idéologique au service des "classes dominantes», du pouvoir, de l'ordre social établi). D'une critique "du premier ordre », il passe à une critique «du second ordre». Nous dirions que la critique de la discipline sociologique d'Ibáñez va plus loin que la critique de Morin, mais les deux critiques s'appuient sur une même critique originaire, commune aux deux : la critique générale du

4" Edgar Morin, Introduction à la pensée complexe, Paris, Seuil, 2005, p. 54.

5" Ibid., p. 57.

51 Voir Edgar Morin, Sociologie, Paris, Fayard, 1984.

52 lbid., p. 10.

5.3 Ibid., p. 35-56. 
paradigme classique dominant et sous-jacent aux disciplines scientifiques particulières, comme la sociologie. Ibáñez, plus sociologue que Morin (qui ne s'est jamais pleinement identifié à ce titre), ne fait qu'épuiser les implications de cette critique dans le domaine de la sociologie, en la reliant à la volonté éthique et politique de changer le système anthroposocial, volonté également commune aux deux auteurs.

Quel est donc le rôle du sociologue dans la société ?, se demande Ibáñez. S'il suit la perspective classique et positiviste, détaché de son "objet d'étude ", il sert au mieux à maintenir la société dans sa forme actuelle, qu'elle soit juste ou injuste. S'il suit la perspective non classique et complexe, rattaché à son "objet d'étude ", il peut servir aussi à projeter la société vers des formes futures possibles, avec la force de l'utopie. Si, déçu par les aberrations historiques commises en invoquant le principe de la Révolution, Morin se contente de chercher la réforme des structures sociales en formulant un nouvel humanisme d'ordre planétaire (modèle de la Terre-Patrie), Ibáñez cherche ouvertement sa révolution en invoquant la substitution du socialisme - de type libertaire, héritier lui-aussi de Mai 68, qui opère une morphogénèse sociale $^{54}$ - à la sociologie en tant que dispositif objectiviste et positiviste, idéologie au service du maintien de l'ordre social. La sociologie socialiste appartiendrait à un ordre logique supérieur à la sociologie sociologique, bien que sa puissance épistémologique ne soit pas non plus illimitée (problème des échecs historiques du socialisme "réel ") $)^{55}$. Morin et Ibáñez dépassent tout au long de leur œuvre les limites de la science (sociologique) en allant au-delà de la sociologie, vers l'horizon de l'utopie, qui permet de se diriger vers un monde plus juste. La sociologie naît avec les révolutions bourgeoises, en pétrifiant précisément les rapports sociaux (la structure) au service des intérêts de la classe bourgeoise. Mais une sociologie socialiste " imaginaire " peut viser le futur, les rapports des rapports, favoriser le changement de la structure.

${ }^{54}$ «Le discours sociologique et le dispositif au service duquel il est tendent à fixer la structure des rapports sociaux, le discours et le dispositif socialiste tendent à transformer la structure en direction de l'un de ses états possibles [...] La sociologie ponctue la structure, le socialisme ponctue le système » (Jesús Ibáñez, Del algoritmo..., op. citt., p. 93-94).

55 L'ensemble des articles qui exposent les vues politiques d'Ibáñez a été rassemblé dans Jesús Ibáñez, A contracorriente, Madrid, Fundamentos, 1997. 
Caminante no bay camino, se bace camino al andar. Tous deux préconisent la mutation du système, mais leur discours diverge dans le ton et dans l'accent qu'ils mettent sur tel ou tel élément.

Morin a construit un paradigme complexe pour la science en général; Ibáñez a construit un paradigme complexe de l'investigation sociale. La perspective dialectique du paradigme complexe d'Ibáñez visait à totaliser l'investigation sociale en intégrant tous les enjeux que nous avons signalés jusqu'à présent (et en intégrant les perspectives qui la précèdent), mais sa mort inattendue, survenue en 1992, ne lui permit pas de mener à bien le développement de sa pensée. La perspective dialectique ignore les limites, atteint le système (la perspective distributive n'atteint que les éléments, la perspective structurale atteint la structure), touche le registre lacanien du réel (la perspective distributive touche le régistre de l'imaginaire en s'aliénant par les limites fantasmatiques des reflets des "données » et des paroles, la perspective structurale touche le registre du symbolique en s'aliénant par les limites langagières du texte "donné "), pense la pensée, introduit un doute généralisé (du second ordre), présente une composante sémiotique (la force de la parole) et tente aussi d'écrire (les perspectives distributive et structurale se limitent à lire le texte du pouvoir). Elle intègre action et réflexion sur l'action, en opérant in vivo et pas in vitro, comme la perspective distributive et la perspective structurale. Elle élimine toute séparation sujet/objet. C'est un appareil de libération ${ }^{56}$. Pendant un moment, Ibáñez fut tenté par la socianalyse et l'analyse institutionnelle de Lapassade et Lourau. Serait-il le modèle parfait de la perspective dialectique? Mai 68 apportait une concrétisation historique illustrant les idées d'lbáñez: dans une session de socianalyse dirigée par Lourau à la Faculté de Sociologie de l'Université de Nanterre, émerge le désir d'occuper le Rectorat ; la socianalyse aboutit à un mouvement révolutionnaire. La perspective dialectique inclut une technique "micro", la socianalyse, et une technique "macro", la révolution. Mai 68 confirma que la technique « micro » pouvait conduire à la technique

56 Jesús Ibáñez, Del algoritmo..., op. cit., p. 205. 
« macro ${ }^{57} »$. Bien que nous ayons oublié aujourd'hui ces techniques révolutionnaires (dans tous les sens du mot), cela ne doit pas nous décourager ; nous devons toujours explorer le champ empirique de la perspective dialectique (qui conserve sa solidité à ce niveau conceptuel) en envisageant de nouvelles techniques de recherche. Différentes modalités de recherche-action-participative s'avèrent toujours comme possibles dans ce domaine, et nous pourrions même envisager une modélisation multi-agents dans le cadre spécifique d'une «modélisation participative " comme le modèle le plus complet d'une technique dialectique/complexe au niveau "micro ». En ce qui concerne la révolution, elle persiste toujours comme horizon utopique de changement du système.

Cependant, les approches de Morin et d'Ibáñez deviennent complémentaires, car Ibáñez a beaucoup travaillé sur la perspective structurale, mais peu sur la perspective dialectique ${ }^{58}$, tandis que Morin a plus développé la perspective dialectique que la perspective structurale : or, le système complexe organisé anthropo-social inclut, outre des éléments, une structure et un système.

\section{Conclusion : pour une dialogique Edgar Morin/Jesús Ibáñez constitutive d'un système d'idées ouvert}

La pensée complexe morinienne serait la pensée propre à la perspective dialectique du sociologue nomade (la perspective dialectique serait l'aboutissement du paradigme complexe et présupposerait les perspectives qui la précèdent) ${ }^{59}$. " La vision complexe (qui inclut l'anamnèse de tout le censuré) est le propre des sociologues de la minorité ou placés dans la perspective de la minorité, dans la perspective

57 Jesús Ibáñez, "Perspectivas de la investigación social : el diseño en las tres perspectivas », Manuel García Ferrando, Jesús Ibáñez et Francisco Alvira (dir.), El análisis de la realidad social. Métodos y técnicas de investigación, Alianza, Madrid, 1986, p. 69.

58 Son ouvrage le plus accompli est $M$ ás alla de la sociologia, qui s'inscrirait dans la perspective structurale. Del algoritmo al sujeto et El regreso del sujeto représentent l'annonce d'une perspective dialectique que la mort l'empêcha de développer.

59 Sur les rapports entre le paradigme de la complexité et la culture logico-philosophique dialectique, voir Lucien Sève, Émergence, complexité et dialectique. Surles systèmes dynamiques non linéaires, Paris, Odile Jacob, 2005. 


\section{NPSS, VOLUME 3, NUMÉRO 2, 2008}

de ceux d'en bas ou des mauvais ${ }^{(1)}$ ". Le terme "minorité » correspond aux dominés, tandis que le terme «majorité » correspond aux dominants (c'est un concept qualitatif plutôt que quantitatif). La sociologie complexe, selon Ibáñez, est toujours une sociologie critique : si des auteurs comme Hayek construisent une théorie économique néo-libérale en s'appuyant sur les théories de la complexité, Ibáñez, en s'appuyant sur les mêmes théories, construit une théorie sociale révolutionnaire. Ibáñez nous fournit une image très révélatrice avec laquelle nous pouvons conclure notre esquisse d'une sociologie complexe ${ }^{61}$. Résumons cette image. Soit un sociologue qui arrive à un carrefour gnoséologique et axiologique (toute science implique une conscience, toute épistémologie implique une éthique) où se croisent un «bon " chemin "vers la droite » et un «mauvais " chemin «vers la gauche» (c'est le champ des oppositions binaires). Ce sociologue adopte une vision complexe s'il doute, et s'il doute sur le doute. Le sociologue sédentaire n'hésite pas et choisit toujours le chemin de droite, un chemin en ordre. Le sociologue nomade hésite et se retrouve empêtré dans les boucles réflexives moriniennes (principe dialogique). Le chemin de droite est toujours une droite, qui est toujours tracée par une règle, la règle du pouvoir (toute droite est artificielle, toute réalité est toujours une Réalité). La liberté face aux contraintes et aux impositions commence avec le doute (du scientifique) face aux carrefours (de la science) : le doute du premier ordre (doute entre les termes de l'opposition) permet la liberté d'une nouvelle lecture (choisir le mauvais chemin), le doute du second ordre (doute sur le doute, entre la bifurcation et la nonbifurcation) permet la liberté d'une nouvelle écriture (effacer les bifurcations et (ou) proposer de nouvelles bifurcations). C'est là le sens ultime de la méthode morinienne, qui équivaut étymologiquement à un méta-chemin. Le paradigme complexe d'Ibáñez converge finalement avec le paradigme complexe de Morin.

Il faut noter qu'il ne s'agit pas d'établir des oppositions. Il ne s'agit pas seulement de choisir le chemin de gauche, mais il ne s'agit surtout pas de choisir le chemin de droite. Il s'agit de douter et de douter sur le doute afin que les oppositions tombent et que l'espace strié du pouvoir

\footnotetext{
al Jesús Ibáñez, Del algoritmo..., op. cit., p. 35.
}

6) Ibid., p. 37. 
devienne l'espace lisse de la liberté. L'espace lisse implique l'infinitude des possibilités (une nouvelle sociologie et une nouvelle société deviennent alors possibles) : le retour nomade au désert du réel face aux emplacements sédentaires dans les mirages imaginaires et dans les constructions symboliques, l'impossible devenant origine de tous les possibles. Il ne s'agit pas de céder à la tentative des oppositions (dans ce cas nous serions toujours dans l'ancien paradigme). Le paradigme de la complexité est le paradigme de la complexité et le paradigme de la simplification. Les trois perspectives de l'investigation sociale (distributive, structurale et dialectique) sont nécessaires et complémentaires (un système anthropo-social inclut des éléments abordés par la perspective distributive, la structure abordée par la perspective structurale et le système abordé par la perspective dialectique). La sociologie complexe ou post-positiviste (nomade et critique, selon les termes d'Ibáñez) inclut la sociologie simplifiée ou positiviste (sédentaire et dogmatique) tout en la dépassant. La sociologie positiviste se limite à tenir compte de l'état actuel du système social (elle est conservatrice, d'une façon consciente ou inconsciente). La sociologie complexe envisagerait, en revanche, outre les états actuels, les états virtuels possibles (elle est utopique, d'une façon consciente) ${ }^{62}$. Selon Ibáñez, la pensée complexe, de la même façon que les nombres complexes en mathématiques, se caractérise par ses composants imaginaires, qui prennent place dans le temps, dans les futurs possibles ${ }^{63}$. La sociologie positiviste envisagerait l'équilibre, la conservation, la statique sociale. La sociologie complexe envisagerait aussi la crise, la réforme, la dynamique sociale. Morin et Ibáñez ont contribué à rendre possible cette sociologie complexe.

Entre Morin et Ibáñez s'établit finalement une dialogique très enrichissante qui surmonte les convergences et divergences initiales pour construire un système d'idées hypercomplexe avec des relations internes d'antagonisme, complémentarité et concurrence, une unitas multiplex. Nous avons signalé au long de cet article les concurrences (en

62 C'est là que réside le sens de nombreux ouvrages de Morin, tels que Introduction à une politique de l'bomme (Paris, Seuil, 1965), Pour sortir du XXe siècle (Paris, Nathan, 1981), Penser l'Europe (Paris, Gallimard, 1987) ou Terre-Patrie (en collaboration avec AnneBrigitte Kern, Paris, Seuil, 1993).

${ }^{63}$ Voir George Spencer Brown, Laws of form, New York, Bantam Books, 1972. 
ontologie et en épistémologie), les antagonismes (au niveau de la forme, du langage) ainsi que les complémentarités (si Ibáñez a développé davantage le niveau de la structure, Morin a développé davantage le niveau du système). C'est ainsi, à partir de ces multiples relations, que nous pouvons construire un système d'idées qui combine les approches de Morin et d'Ibáñez, un système caractérisé par le principe complexe fondamental de la dialogique. Mais ce système doit être un système ouvert et non pas un système fermé.

C'est là que nous devons faire une importante remarque. Les travaux de Morin et d'Ibáñez restent à un niveau théorique. Or, le paradigme de la complexité ne peut pas être réduit à une philosophie de la complexité. En suivant les trois niveaux verticaux du paradigme complexe d'Ibáñez, nous constatons qu'il nous manque une méthodologie, ainsi qu'une technologie propres. Le système d'idées Morin/Ibáñez doit s'ouvrir à de nouvelles méthodologies, à de nouvelles technologies du «bruit» externe qui pourrait l'aider à se complexifier et à mieux s'adapter au milieu scientifique existant (pour pouvoir ainsi le révolutionner?).

Ceci est source de problèmes, mais aussi d'enjeux. En mobilisant les trois niveaux verticaux, nous dirions que la pensée complexe morinienne s'inscrit dans le troisième niveau (niveau épistémologique) et non pas dans le deuxième (niveau méthodologique). La perspective dialectique qui s'articule avec le niveau épistémologique atteint le système, le côté le plus large de l'unité et de la globalité. La Méthode morinienne est plutôt une pré-Méthode ${ }^{64}$. Il est possible de faire le même reproche à la «pensée complexe » d'Ibáñez. La pensée complexe devient très révélatrice au niveau épistémologique - ainsi qu'au niveau ontologique -, mais pas si évidente au niveau méthodologique. Nous devrions chercher davantage dans les ouvrages antérieurs de Morin, comme La métamorphose de Plodémet ou La rumeur d'Orléans, afin de trouver des études plus empiriques et des méthodologies plus précises (qui

(n. J'emprunte le terme à Jacques Ardoino (Séminaire Savoir, Connaissance, Éducation, en collaboration avec Alfredo Pena-Vega, EHESS, Paris, 2005-2006). Pascal Roggero défend une position similaire dans la conclusion de l'ouvrage qu'il a dirigé $L a$ complexité territoriale : entre processus et projets, Paris, L'Harmattan, coll. "Ingénium ", 2006. I.es travaux du Centre Interdisciplinaire d'lutude et de Recherche sur les Systèmes Sociaux (CIRESS) de l'U niversité de Toulouse I s'inscrivent également dans cette perspective de traduction méthodologique de la pensée complexe. 
configurent ce qu'il appelle la "sociologie du présent ", ainsi que l'approche dite « interdisciplinaire »). En tout cas, La Méthode peut rester un traité ontologique et épistémologique fondamental, auquel nous pourrions adjoindre les propositions du «premier » Morin, même si cela n'est pas non plus exempt de controverses (une lecture «complexe»du "premier " Morin ne serait-elle pas finalement une reconstruction ad boc ?). En ce qui concerne Ibáñez, nous disposons de ses travaux sur le "groupe de discussion », mais sa forte critique envers ce modèle de recherche ${ }^{65}$, considéré comme un instrument d'exploitation du capitalisme de consommation, l'invalide comme méthodologie propre à une sociologie complexe. En ce qui concerne la « socianalyse», elle ne fut que peu développée par lui.

Le paradigme scientifique de la complexité requiert aussi des propositions propres en ce qui concerne les différentes techniques de recherche. Nous avons besoin d'une technologie de la complexité. Dans ce sens, des initiatives dans le champ de ce que Morin appelle la " complexité restreinte " sont prometteuses, dans la mesure où elles seraient bien capables de s'harmoniser avec une "complexité généralisée ", plus spéculative. C'est l'approche dite des "systèmes complexes ", qui cherche à modéliser les phénomènes d'émergence dont les modèles analytiques ne savent pas rendre compte. En utilisant des techniques informatiques innovatrices et puissantes, telles que notamment les automates cellulaires et, plus encore, les systèmes multiagents, elle trouve des applications dans les sciences sociales à partir du champ appelé "intelligence artificielle distribuée ". La référence de premier ordre au niveau de la recherche en systèmes complexes est le Santa Fe Institute, aux États-Unis. Parmi les nombreux travaux dans ce domaine, nous pouvons citer le réseau thématique Sociologie et systèmes complexes, de l'Association Française de Sociologie (AFS), spécialement consacré à la modélisation de systèmes sociaux complexes. Cependant, pour le moment, nous devons garder un esprit critique à l'égard d'une possible intégration de la complexité restreinte et la complexité généralisée, même si elle est nécessaire : selon Morin, la

¿s Cette critique forme le noyau central de son ouvrage Más allá de la sociologia. 
complexité restreinte agit toujours sous le signe du paradigme classique de simplification ${ }^{66}$.

Le système Morin/Ibáñez va au-delà des limites dictées par le scientisme. Outre la chaîne ontologie / épistémologie / méthodologie / technologie, nous avons vu comment une dimension ultérieure s'ouvre lors de l'application à la sociologie des théories de la complexité, une dimension qui articule la science avec la conscience, qui fait correspondre une pragmatique explicite ou implicite à la sémantique. La pensée complexe est aussi susceptible de devenir une post-Méthode ${ }^{67}$. C'est le domaine de l'au-delà de la sociologie, où se dresse l'u-topos, le non-lieu, l'utopie, qui sert à marcher, vers un horizon plus juste. Des initiatives en éducation et en politique sont déjà envisagées. Morin et Ibáñez produiront au cours de leur carrière de nombreux atticles et livres à la lumière de l'actualité la plus récente (et toujours politique), avec courage, sans peur. En payant aussi le prix que la volonté de faire face au pouvoir suppose : Morin fut soumis à des jugements et des menaces en raison d'un article sur le sort des Palestiniens ; Ibáñez apparut sur la liste des individus qui devaient être exécutés immédiatement en cas de triomphe du Coup d'État en Espagne en 1981. Le nomade sort des terrains sûrs et évidents pour s'aventurer dans le désert incertain plein de risques. Il illustre alors ce qu'Anthony Wilden appelle une « rhétorique de guérilla " ${ }^{60}$ " face au couple savoir/pouvoir dominant qui impose une idéologie, qui tel un continent, possède une structure fixe, inamovible : il mobilise un discours d'un ordre logique supérieur qui atteint les plus hauts sommets du contexte. L'utopie est certes une île, mais un ensemble d'iles peut former un archipel : l'archipel peut faire bouger la structure. C'est là que réside l'espoir. Le Vaisseau Spatial Terre peut encore se sauver ${ }^{69}$.

o6 Edgar Morin, “ Complexité restreinte... », op. cit., p. 49.

67 J'emprunte le terme à Claude Fischler (communication personnelle).

68 Anthony Wilden, System and Structure..., op. cit., p. 58.

69 «La porte est ouverte donc sur l'improbable, même si l'accroissement mondial de barbarie le rend actuellement inconcevable. Paradoxalement, le chaos où l'hum anité risque de sombrer porte en lui son ultime chance. Pourquoi? Tout d'abord parce que la proximité du danger favorise les prises de conscience, qui peuvent alors se multiplier, s'amplifier et faire surgir une grande politique de salut terrestre. Et surtout pour la raison suivante : quand un système est incapable de traiter ses problèmes vitaux, 
Rappelons pour finir ce que Morin écrivait en 1974 dans la revue Communications : "cela peut chagriner beaucoup de reconnaitre que s'il existe des sociologues, la sociologie n'existe pas encore ${ }^{70}$ ". Nous devrions alors nous demander : avons-nous vraiment avancé depuis plus de trente ans? Il semblerait que le paradigme dominant soit toujours le même, malgré les apports de Morin et d'Ibáñez. Mais la science, plus changeante certes que la théologie (Whitehead), peut toujours se réinventer. Pourrons-nous surmonter le paradigme de la simplification et atteindre le paradigme de la complexité ? Nous ne saurions répondre sans hésitation à la question proposée : nomades nous-mêmes, apprentis nomades, nous doutons et nous doutons sur le doute, l'incertitude nous envahit, notre propre pensée se maintient à la périlleuse " température de sa propre destruction" (Morin), l'égarement dans le labyrinthe est plus que probable, mais la sortie victorieuse et la conquête du continent l'est aussi.

Nous sommes partis des orientations proposées par deux auteurs nomades. Il s'agit à présent de commencer à construire en marchant le chemin d'une sociologie complexe. Caminante no hay camino, se bace camino al andar...

\section{Bibliographie}

Castoriadis, Cornelius, L'institution imaginaire de la societé, Paris, Seuil, 1975.

Deleuze, Gilles et Félix Guattari, Capitalisme et schizopbrénie: mille plateaux, Paris, Minuit, 1980.

Dumouchel, Paul et Jean-Pierre Dupuy, L'auto-organisation : de la physique au politique (actes du Colloque de Cerisy, 10-17 juin 1981), Paris, Seuil, 1983.

Dupuy, Jean-Pierre, "Les techniques de l'auto-organisation ", Comelius Castoriadis (1922-1997). Réinventer l'autonomie, $1^{\mathrm{er}}$ mars 2007, Université Paris VIII.

Dupuy, Jean-Pierre, Aux origines des sciences cognitives, Paris, La Découverte, 1994.

Dupuy, Jean-Pierre, Introduction aux sciences sociales. L-ogique des phénomènes collectifs, Paris, Ellipses, 1992.

soit il se désintègre, soit il est capable, dans sa désintégration même, de se métamorphoser en un système plus riche, capable de traiter ses problèmes" (Edgar Morin, "Vers l'abime?", Le Monde, $1^{\text {er }}$ janvier 2003).

70 Edgar Morin, «La nature de la société ", Communications, 22, Paris, EHESS-Seuil, 1974, p. 31. 
Foerster, Heinz von, "On Self-Organizing Systems and their Environments", dans Marshall. C. Yovitts et Scott Cameron (dir.), Self-Organizing Systems, New York, Pergamon Press, 1960, p. 31-50.

Gilbert, Nigel et Klaus G. Troitzsch, Simulation for the Social Scientist, Open University Press, McGraw-Hill, 2005.

Ibáñez, Jesús, A contracorriente, Madrid, Fiundamentos, 1997.

I báñez, Jesús, El regreso del sujeto. L a investigación social de segundo orden, Madrid, siglo XXI, 1994.

Ibáñez, Jesús (coord.), Nuevos avances en la investigación social I. I a investigación social de segundo orden, Barcelona, Proyecto A Ediciones, Barcelona, 1988.

Ibáñez, Jesús, "Perspectivas de la investigación social : el diseño en las tres perspectivas ", dans Ferrando Manuel García, Jesús Ibáñez et I'rancisco Alvira (dir.), El anuilisis de la realidad sorial. Métodos y técnicus de investigación, Alianza, Madrid, 1986, p. 49-83.

Ibáñez, Jesús, Del algoritmo al sujeto. Perppectivas de la investigación social, Madrid, siglo XXI, 1985.

Ibáñez, Jesús, "Las medidas de la sociedad ", Revista Española de Investigaciones Suciologicas, 29, 1985, p. 85-127.

Ibánez, Jesús, Mais allá de la sociologia. El grupo de discusión : técnica y critica, Madrid, siglo XXI, 1979.

Le Moigne, Jean-Louis, "Complexité ", dans Dominique Lecourt, Dictionnaire d'bistoire et philosopbie des sciences, Paris, PUF, 2006, p. 242.

Le Moigne, Jean-Louis, La modélisation des systèmes complexes, Paris, Dunod, 1990.

Morin, Edgat, "Complexité restreinte, complexité générale ", dans Le Moigne, JeanLouis et Edgar Morin, Intelligence de la complexité. Épistémologie et pragmatique, Paris, Éditions de l'Aube, 2007, p. 28-64.

Morin, Edgar, Introduction à la pensée complexe, Paris, Seuil, 2005.

Morin, Edgar, "Vers l'abîme?", Le Monde, 1" janvier 2003.

Morin, Edgar, La Métbude V. L'bumanité de l'bumanité, Paris, Seuil, 2001.

Morin, Fdgar, La Méthode IV. Lees idées, Paris, Seuil, 1990.

Morin, Edgar, Scciologie, Paris, Fayard, 1984.

Morin, Edgar, La Methode II. La vie de la vie, Paris, Seuil, 1980.

Morin, Edgar, La Méthode I. La nature de la nature, Paris, Seuil, 1977.

Morin, Edgar, «La nature de la société », Communications, 22, Paris, EHESS-Seuil, 1974, p. 3-31.

Morin, Edgar, Le paradigme perdu : la nature bumaine, Paris, Seuil, 1973

Morin, Ldgar, "L'événement-sphynx », Communications, 18, 1972, p. 173-192.

Roggero, Pascal, "Pour une sociologie d'après La Méthode », Communications, ${ }^{\circ} 82$, Paris, EHESS-Seuil, 2008, p. 143-159.

Roggero, Pascal (dir.), Ia complexité territoriale : entre processus et projets, Paris, L’Harmattan, coll. "Ingénium », 2006.

Roggero, Pascal et Christophe Sibertin-Blanc, "Préambule 》, Nouvelles perspectives en sciences sociales, vol. $2, \mathrm{n}^{\circ} 2$, Ontario, Prise de parole, 2008.

Sève, Lucien, Émergence, complexité et dialectique. Sur les systèmes dynamiques non linéaires, Paris, Odile Jacob, 2005. 
Spencer Brown, George, Law's of form, New York, Bantam Books, 1972.

Watzlawick, Paul (dir.), L'invention de la réalité. Contributions au constructivisme, Paris, Seuil, 1988.

Wilden, Anthony, «Context Theory: The New Science », SRI 5/2, 1985, p. 97-116.

Wilden, Anthony, System and Structure. Essays in Communication and Exchange, Londres, Tavistock, 1972. 